\title{
COHOMOLOGY OF LIE TRIPLE SYSTEMS AND LIE ALGEBRAS WITH INVOLUTION( $\left.{ }^{1}\right)$
}

\author{
BY \\ B. HARRIS
}

A Lie triple system is a subspace of a Lie algebra closed under the ternary composition $[[x y] z]$; equivalently, it may be defined as the subspace of elements mapped into their negatives by an automorphism of order two (an involution) in a Lie algebra; or, finally, a Lie triple system may be defined by a set of identities.

Lie triple systems were first noted by E. Cartan in his studies on totally geodesic submanifolds of Lie groups and on symmetric spaces [1] (see also $[10])$ : Lie triple systems are related to totally geodesic submanifolds in the same way that Lie algebras are related to analytic subgroups, and the symmetry in a symmetric space gives rise to the involution in the Lie algebra.

Lie triple systems were studied from the algebraic point of view by Jacobson $[6 ; 7]$ and Lister $[9]$, the latter giving a complete structure theory including the classification of the simple finite-dimensional systems (in characteristic zero), the Levi decomposition, and (a special case of) the first Whitehead lemma. Simpler axioms were given by Yamaguti [14], who has also studied these and more general systems $[15 ; 16]$.

In this paper we introduce cohomology groups for Lie triple systems and show that the usual interpretations (in terms of derivations and factor sets) hold for the first and second cohomology groups. In particular we obtain the two Whitehead lemmas (in characteristic zero). Further, these cohomology groups fit into the theory of [2]: they are the cohomology groups of a supplemented associative algebra (very closely related to the universal associative algebra of the Lie triple system).

The groups may briefly be described as follows: if $T$ is any Lie triple system and $M$ a $T$-module we can construct the universal Lie algebra $L_{u}(T)$ of $T$ and the "standard extension" $M_{s}$ which is an $L_{u}(T)$ module; an involution $\sigma$ operates on both $L_{u}(T)$ and $M_{s}$ such that the elements mapped into their negatives are $T$ and $M$ respectively, and $\sigma([l, n])=[\sigma(l), \sigma(n)]$ for $l \in L_{u}(T), n \in M_{\varepsilon}$. Now (in a more general situation) if $L$ is any Lie algebra with involution $\sigma$ and $N$ any $L$-module also with involution $\sigma$, then $\sigma$ also operates on the cohomology groups $H^{n}(L, N)=\operatorname{Ext}_{U}^{n}(\Phi, N)(\Phi$ the base field, $U$ the universal associative algebra of $L$ ); we assume further that the characteristic is not 2, so that $H^{n}(L, N)$ is a direct sum of two subspaces invariant under $\sigma$ : say $H_{+}^{n}(L, N), H_{-}^{n}(L, N)$. We show that $H_{+}^{n}(L, N)=\operatorname{Ext}_{R}^{n}(\Phi, N)$

Presented to the Society, April 16, 1960, under the title Cohomology of Lie triple systems; received by the editors August 24, 1959.

(1) This work was supported in part by a grant from the National Science Foundation. 
where $K$ is the algebra $U+U \sigma$ (with multiplication rules $\sigma \cdot l=\sigma(l) \sigma$ for $l \in L$, $\left.\sigma^{2}=1\right), \sigma$ operates as the identity on $\Phi$, and there exists a supplementation of $K$ onto $\Phi$. Coming back to $T$ and the $T$-module $M$, we define the $n$th cohomology group of $T$ with coefficients in $M, H^{n}(T, M)$, as $H_{+}^{n}\left(L_{u}(T), M_{s}\right)$ $=\operatorname{Ext}_{K}^{n}\left(\Phi, M_{s}\right)$. The $n$-cochains we are considering are thus the multilinear alternating functions $f$ of $n$ variables from $L_{u}(T)$ to $M_{3}$ satisfying $f\left(\sigma\left(x_{1}\right), \cdots, \sigma\left(x_{n}\right)\right)=\sigma f\left(x_{1}, \cdots, x_{n}\right)$. In particular, we may take the trivial $T$-module $M=\Phi$ : then $M_{s}=\Phi$ also but with $\sigma$ acting as -1 , and the cochains satisfy $f\left(\sigma\left(x_{1}\right), \cdots, \sigma\left(x_{n}\right)\right)=-f\left(x_{1}, \cdots, x_{n}\right)$.

A similar construction of cohomology groups for associative algebras with an anti-automorphism of period two was given in [12]. The analogue for twosided modules with involution of the cross-product algebra $U+U \sigma$ is used in [5], and the theory of extensions of algebras with a group of operators is given in [13].

1. Basic definitions. In this section we recall the basic definitions of Lie triple systems and their enveloping Lie and associative algebras [7;9] and, following the general idea of [4], define modules for Lie triple systems. All algebras, modules, etc. will be assumed to be vector spaces over a field $\Phi$ of characteristic different from two; all mappings will be assumed linear over $\Phi$.

A Lie triple system $T$ is a vector space over $\Phi$ with a ternary composition $[a b c]$ which is trilinear and satisfies

$$
\begin{gathered}
{[a a b]=0,} \\
{[a b c]+[b c a]+[c a b]=0,} \\
{[a b[x y z]]=[[a b x] y z]+[x[a b y] z]+[x y[a b z]] .}
\end{gathered}
$$

Equation (1.3) says that the map $x \rightarrow[a b x]$ is a Lie triple system derivation.

We will not actually use these identities but use only the fact that they exist.

If $L$ is a Lie algebra, with product $[a b]$, then the ternary composition $[a b c]=[[a b] c]$ satisfies the above identities. Conversely, it is shown in [14] or [7] that any Lie triple system $T$ may be considered as a subspace of a Lie algebra $L$ in such a way that $[a b c]=[[a b] c]$. The construction is as follows: let $L$ be the vector space direct sum $T \oplus(T \otimes T)^{-}$, where $(T \otimes T)^{-}$is the factor space of $(T \otimes T)^{-}$(plain $\otimes$ denoting tensor product over $\left.\Phi\right)$ modulo the subspace of all $\sum a_{i} \otimes b_{i}$ such that $\sum\left[a_{i} b_{i} x\right]=0$ for all $x$ in $T$. The product $[x y]$ in $L$ is defined by:

$$
\begin{aligned}
{[a b] } & =(a \otimes b)^{-}(a, b \text { in } T), \\
{\left[\left(\sum\left[a_{i} b_{i}\right]\right) c\right] } & =\sum\left[a_{i} b_{i} c\right] \\
{\left[c\left(\sum\left[a_{i} b_{i}\right]\right)\right] } & =-\sum\left[a_{i} b_{i} c\right] \\
{\left[\left(\sum\left[a_{i} b_{i}\right]\right)\left(\sum\left[c_{i} d_{i}\right]\right)\right] } & =\sum\left[\left[a_{i} b_{i} c_{i}\right] d_{i}\right]-\left[\left[a_{i} b_{i} d_{i}\right] c_{i}\right]
\end{aligned}
$$

for $a, b, c, d \in T$. 
$L$ is then a Lie algebra, called the standard enveloping Lie algebra of $T$, and denoted by $L_{0}(T)$ or merely $L_{0}$. It is clear that the natural map of $T$ into $L_{s}(T)$ is $1-1$ and $L_{s}(T)=T \oplus[T, T]$ as vector space.

One can also construct a universal enveloping Lie algebra $L_{u}(T)$, with the property that any homomorphism of $T$ into a Lie algebra extends to a homomorphism of $L_{u}(T)$, and a universal associative algebra (always with identity) $U(T)$ with the same property relative to homomorphisms of $T$ into associative algebras. $U(T)$ is then also the universal associative algebra of $L_{u}(T)$, the natural maps of $T$ into $U(T)$ and $L_{u}(T)$ are 1-1, and $L_{u}(T)=T \oplus[T, T]$.

From the above remarks we see that a Lie triple system may also be defined as a subspace of a Lie algebra closed under $[[a b] c]$. Most useful for us, however, is the following definition: There exists an automorphism $\sigma$ of period 2 (i.e., $\sigma^{2}=$ identity map) in a Lie algebra $L$ such that $T=\{l \in L \mid \sigma(l)=-l\}$. In $L_{u}(T)=T \oplus[T, T]$ the automorphism is: $\sigma(t)=-t, \sigma\left(\left[t_{1} t_{2}\right]\right)=\left[t_{1} t_{2}\right]$ for $t, t_{i}$ in $T . \sigma$ will be called the involution.

A vector space $M$ is called a module for the Lie triple system $T$ if the vector space direct sum $E=T \oplus M$ is itself a Lie triple system in such a way that: (1) $T$ is a subsystem, (2) $[a b c]$ lies in $M$ if any one of $a, b, c$ is in $M$, (3) [abc] $=0$ if any two of $a, b, c$ are in $M$. Equivalently, a module $M$ is a vector space together with a map of $M \otimes T \otimes T$ into $M$ satisfying the identities (1.1)-(1.3) (and condition (3) above). $E=T \oplus M$ will be called the semi-direct sum, or split null extension, or inessential extension, of $T$ by $M$.

We may also describe a Lie triple system module $M$ in terms of a Lie algebra module and an involution. For this purpose we define the standard extension $M_{s}=N_{s}(M)$, which will be a Lie algebra module over $L_{u}(T)$ : let $N=N_{8}(M)$ be the vector space direct sum $M \oplus(M \otimes T)^{-}$where $(M \otimes T)^{-}$is the quotient space $M \otimes T$ modulo the subspace $\left\{\sum m_{i} \otimes t_{i} \mid \sum\left[m_{i}, t_{i}, t\right]=0\right.$ for all $t \in T\}$. The rules giving the structure of $N_{s}(M)$ and $L_{u}(T)$ module are the obvious analogues of (1.4); to show these rules are well defined we proceed as follows: first form the semi-direct sum $E=T \oplus M$ and its standard Lie algebra (see [7]) $L_{s}(E)=E \oplus(E \otimes E)^{-}$. The natural injection of $M \otimes T$ into $E \otimes E$ gives an injection of $(M \otimes T)^{-}$into $(E \otimes E)^{-}$(the conditions that $\sum\left(m_{i} \otimes t_{i}\right)$ be zero in $(M \otimes T)^{-}$or $(E \otimes E)^{-}$are the same, namely $\sum\left[m_{i}, t_{i}, t\right]$ $=0$ in $M$ for all $t \in T$, since $\sum\left[m_{i}, t_{i}, m\right]=0$ automatically for $m \in M$ ). Thus $N_{s}(M)$ may be considered as a subspace of $L_{s}(E)$ and is in fact an ideal in $L_{s}(E)$. Since $L_{s}(E)$ is a Lie algebra containing the Lie triple system $T$, the identity map $T \rightarrow T \subset L_{s}(E)$ may be extended to a homomorphism of $L_{u}(T)$ into $L_{s}(E): N_{s}(M)$ being an ideal in $L_{s}(E)$, then becomes also an $L_{u}(T)$ module.

Now define the involution $\sigma$ in $L_{s}(E)$ by $\sigma(x)=-x$ for $x \in E, \sigma(x)=x$ for $x \in[E, E]$. Then $\sigma$ maps $N_{\bullet}(M)$ into itself and $\sigma(m)=-m, \sigma([t, m])=[t, m]$ for $m \in M, t \in T$. It is then clear that $\sigma([l, n])=[\sigma(l), \sigma(n)]$ for $l \in L_{u}(T)$, $n \in N_{s}(T)$ and that $M=\left\{n \in N_{\bullet}(T) \mid \sigma(n)=-n\right\}$. 
The module $N_{s}(T)$ of $L_{u}(T)$ may also be considered as a module over $U(T)$, which we will write as a left module by: $x \cdot n=[x, n]=-[n, x]$ for $x \in L_{u}(T)$ $\subset U(T)$. The automorphism $\sigma$ of $L_{u}(T)$ induces an automorphism, also denoted by $\sigma$, of period 2 in $U(T)$, and

$$
\sigma(u \cdot n)=\sigma(u) \cdot \sigma(n), \quad \text { for } u \in U(T), n \in N_{\imath}(T) .
$$

In general, if $N$ is a left module for an associative algebra $U$, or a Lie algebra $L$, and there exists a 1-1 map $\sigma$ of $N$ onto itself, and an involution, also denoted by $\sigma$, in $U$, or in $L$, satisfying (1.5), $N$ is called a module with involution. Equivalently, a left $U$-module with involution is a left module over an algebra $K$ constructed as follows: as vector space $K=U \oplus U$, and the multiplication is

$$
(a \oplus b)(c \oplus d)=(a c+b \sigma(d)) \oplus(a d+b \sigma(c)) .
$$

We shall write $a+b \sigma$ for $a \oplus b$, so that the multiplication rules in $K$ are: $\sigma a=a \sigma(a), \sigma^{2}=1$; we shall write $U+U \sigma$ for $K$. For $n \in N$, we shall write $\sigma \cdot n$ for $\sigma(n)$ : thus if $N$ is a left $U$-module with involution, this rule makes $N$ a left $K$-module, while if $N$ is a left $K$-module it is also a left $U$-module with the involution $\sigma$.

If $T$ is a Lie triple system, $M$ a module for $T$, then in general there are many Lie algebra modules with involution $N$ for $L_{u}(T)$ such that $N=M$ $\oplus[T, M]$ (vector space direct sum) and $M=\{n \in N \mid \sigma(n)=-n\}$. Among these the standard extension $N_{s}(M)$ may be characterized by the fact that the identity map $M \rightarrow M$ extends to a homomorphism of $N$ onto $N_{s}(M)$ (for the map $m+\sum\left[t_{i}, m_{i}\right] \rightarrow m+\sum\left(t_{i} \otimes m_{i}\right)^{-}$of $N$ into $N_{s}(M)$ is well defined, and clearly a homomorphism onto).

In the same way the standard extension $L_{s}(T)$ and the universal extension $L_{u}(T)$ may be characterized among all enveloping Lie algebras $L$ of $T$ that satisfy $L=T \oplus[T, T]$ : There is a (unique) homomorphism of $L_{u}(T)$ onto $L$ and one of $L$ onto $L_{s}(T)$ which are the identity on $T$. In particular there is a (unique) homomorphism of $L_{u}(T)$ on $L_{s}(T)$ which is the identity on $T$ : its kernel is the intersection of the center of $L_{u}(T)$ with $[T, T]$. Thus if $L_{u}(T)$ has no center, then $L_{u}(T)$ is isomorphic to $L_{s}(T)$.

These constructions have the following functorial properties:

1. If $M, M^{\prime}$ are modules for the Lie triple system $T$, and $\phi$ is a module homomorphism of $M$ into $M^{\prime}$, then $\phi$ induces a homomorphism $\phi_{s}$ of the $L_{u}(T)$ module $M_{s}=N_{s}(M)$ into $M_{s}^{\prime}$. If $\phi$ is $1-1$ or onto, then $\phi_{s}$ is $1-1$ or onto, respectively.

2. If $T, T^{\prime}$ are Lie triple systems and $\phi$ a homomorphism of $T$ into $T^{\prime}$, then $\phi$ induces a homomorphism $\phi_{u}$ of $L_{u}(T)$ into $L_{u}\left(T^{\prime}\right)$, which is onto if $\phi$ is onto. Further, if $\phi$ is onto, it induces a homomorphism $\phi_{s}$ of $L_{8}(T)$ onto $L_{8}\left(T^{\prime}\right)$.

The above homomorphisms all commute with the involution $\sigma$.

2. Cohomology of Lie algebras with involution. In this section we consider 
Lie algebras $L$ with involution $\sigma$, and $L$-modules $N$ with involution, and study the effect of $\sigma$ on the cohomology groups of $L$ with coefficients in $N$. In the next section we will apply these results to $L=L_{u}(T), T$ a Lie triple system, and $N=M_{2}=N_{s}(M), M$ a $T$-module.

The cohomology groups $H^{n}(L, N)$ of $L$ with coefficients in $N$ are defined [2, Chapter 13] as $\operatorname{Ext}_{U}^{n}(\Phi, N)$ where $U=U(L)$ is the universal enveloping associative algebra of $L$, the structure of the base field $\Phi$ as $U$-module being given by a supplementation $\epsilon: U \rightarrow \Phi$. We also have present the involution $\sigma$ so that we can form $K=U+U \sigma$ : we shall show that $K$ has some of the important properties of $U$, and will study the effect of replacing $U$ to $K$.

The properties of $U$ we shall consider are [2, Chapters 10,13]:

1. The supplementarion $\epsilon: U \rightarrow \Phi$.

2. A homomorphism $E$ of $U$ into $U^{e}=U \otimes U^{*}$ ( $U^{*}$ being the algebra antiisomorphic to $U$ under the map $u \rightarrow u^{*}$ ) such that

$$
\begin{array}{cl}
U \stackrel{\epsilon}{\rightarrow} \Phi \\
E \downarrow & \downarrow \eta \\
U^{\bullet} \underset{\rho}{\rightarrow} & U
\end{array}
$$

is a commutative diagram ( $\eta$ denoting the map $\alpha \rightarrow \alpha 1, \rho$ the map $a \otimes b^{*} \rightarrow a b$ ), i.e. $E(I) \subset J, I$ being the kernel of $\epsilon$ and $J$ that of $\rho$.

3. $J=U^{e} \cdot E(I)$ and $U^{e}$ is a projective right $U$ module ( $U^{e}$ being a right $U$-module under the map $\left(a \otimes b^{*}\right) \cdot c=\left(a \otimes b^{*}\right) E(c)$ for $\left.c \in U\right)$ so that [2, Theorem 10.6.1], if we make a left $U^{e}$-module $N$ into a left $U$-module ${ }_{E} N$ by the map $E$, then $\operatorname{Ext}_{U}^{n}\left(\Phi,{ }_{E} N\right)$ is isomorphic to $\operatorname{Ext}_{U^{n}}^{n}(U, N)$.

4. There is an "antipodism" (isomorphism) $\omega$ of $U$ with $U^{*}$ so that $D=\omega E: U \rightarrow^{E} U \otimes U^{*} \rightarrow{ }^{\otimes}{ }^{\infty} U \otimes U$ is a homomorphism (actually an isomorphism), called a diagonal map.

Since $U$ is a subalgebra of $K$, and $U^{e}$ of $K^{e}=K \otimes K^{*}$, we shall use the same letters as above for the maps involving $K$. We define the supplementation $\epsilon$ of $K$ onto $\Phi$ by $\epsilon(\sigma)=1$, and $\epsilon$ as before on $U$, i.e., $\epsilon(a+b \sigma)=\epsilon(a)+\epsilon(b)$. The kernel $I_{0}$ then contains the kernel $I$ of the restriction of $\epsilon$ to $U$ : in fact $I_{0}=I+I \sigma+\Phi(1-\sigma)$. Since $\sigma(I)=I$ in $U$ and $U=\Phi 1+I, 0$ it is clear that $\epsilon$ is a homomorphism of $K$ onto $\Phi$.

The map $E$ of $U$ into $U^{e}$ will be extended to a map $K \rightarrow K^{e}$ by setting $E(\sigma)=\sigma \otimes \sigma^{*}$ : i.e., we let $E(a+b \sigma)=E(a)+E(b)\left(\sigma \otimes \sigma^{*}\right)$. For $x \in L \subset U$, $E(x)=x \otimes 1-1 \otimes x^{*}$. It is easy to check that $E(\sigma) E(x)=E(\sigma x)$ for $x \in L$, and since $L$ and 1 generate $U, E(\sigma) E(a)=E(\sigma a)$ for all $a \in U$. Finally, $E$ is a homomorphism. $E$ is $1-1$ since the homomorphism $1 \otimes \epsilon^{*}: K \otimes K^{*} \rightarrow K$ by $\left(1 \otimes \epsilon^{*}\right)\left(a \otimes b^{*}\right)=a \epsilon(b)$ satisfies $\left(1 \otimes \epsilon^{*}\right) E(x)=x,\left(1 \otimes \epsilon^{*}\right) E(\sigma)=\sigma,(x \in L)$ and so $\left(1 \otimes \epsilon^{*}\right) E(k)=k$ for $k \in K$.

The kernel $J_{0}$ of the map $K^{e} \rightarrow K$ is the left ideal in $K^{e}$ generated by the 
elements $\left(k \otimes 1-1 \otimes k^{*}\right)$ and so $J_{0}$ contains $J$, the kernel of the map $U^{e} \rightarrow U$. We show first that $J_{0}=K^{e} \cdot E\left(I_{0}\right): I_{0}=I+I \sigma+\Phi(1-\sigma)=I+\sigma I+\Phi(1-\sigma)$; it is known that $E(I) \subset J \subset J_{0}$, thus $E(\sigma I)=E(\sigma) E(I) \subset J_{0}$ also, since $J_{0}$ is a left ideal, and finally $E(1-\sigma)=1 \otimes 1^{*}-\sigma \otimes \sigma^{*}=(\sigma \otimes 1)\left(\sigma \otimes 1^{*}-1 \otimes \sigma^{*}\right) \in J$. Thus $E\left(I_{0}\right) \subset J_{0}$ and $J_{0} \supset K^{e} \cdot E\left(I_{0}\right)$. Conversely, we have to show $k \otimes 1$ $-1 \otimes k^{*} \in K^{e} \cdot E\left(I_{0}\right)$ for $k \in K$. Let $k=a+b \sigma, k \otimes 1-1 \otimes k^{*}=\left(a \otimes 1-1 \otimes a^{*}\right)$ $+\left(b \sigma \otimes 1-1 \otimes(b \sigma)^{*}\right) .\left(a \otimes 1-1 \otimes a^{*}\right) \in J=U^{\bullet} \cdot E(I) \subset K^{\bullet} \cdot E\left(I_{0}\right)$. $\left(b \sigma \otimes 1-1 \otimes(b \sigma)^{*}\right)=\left(b \sigma \otimes 1-1 \otimes \sigma^{*} b^{*}\right)=(b \otimes 1)\left(\sigma \otimes 1-1 \otimes \sigma^{*}\right)$ $+\left(1 \otimes \sigma^{*}\right)\left(b \otimes 1-1 \otimes b^{*}\right)$. Since $\left(\sigma \otimes 1-1 \otimes \sigma^{*}\right)=(\sigma \otimes 1) E(1-\sigma) \in K^{e} E\left(I_{0}\right)$, $k \otimes 1-1 \otimes k^{*} \in K^{e} \cdot E\left(I_{0}\right)$ also. Next, $K_{E}^{e}$ is right $K$-projective ( $K_{B}^{e}$ denoting $K^{e}$ as right $K$-module under the map $E$ ): to show this we first define the isomorphism $\omega$ of $K$ and $K^{*}$ by $\omega(\sigma)=\sigma^{*}$ and, on $U$, (if $x_{i} \in L$ ), $\omega\left(x_{1} \cdots x_{p}\right.$ ) $=(-1)^{p}\left(x_{p}^{*} \cdots x_{1}^{*}\right)$ so that $\omega(a+b \sigma)=\omega(a)+\omega(b) \omega(\sigma)$, so that $D=(1 \otimes \omega) E$ is an isomorphism of $K$ into $K \otimes K$. $\left((1 \otimes \omega)\left(a \otimes b^{*}\right)=a \otimes \omega\left(b^{*}\right)\right)$. Then $K_{E}^{e}$ as right $K$-module is isomorphic to $(K \otimes K)_{D}$ and $D(x)=x \otimes 1+1 \otimes x$ for $x \in L$, $D(\sigma)=\sigma \otimes \sigma .(U \otimes U)_{D}$ is a free right $U$-module [2, Chapter 13, Proposition 4.1] with a basis consisting of monomials $m_{i}=\left(x_{1} \otimes 1\right) \cdots\left(x_{n} \otimes 1\right),\left\{x_{i}\right\}$ a basis for $L$ (since $U \otimes U=U(L \oplus L)$ and the subalgebra $\{x \otimes 1+1 \otimes x\}$ of $L \oplus L$ has a basis of the form $\left\{x_{i} \otimes 1+1 \otimes x_{i}\right\}$, while these elements together with the $\left\{x_{i} \otimes 1\right\}$ form a basis for $\left.L \oplus L\right)$. In fact we may also assume that $x_{i}^{\sigma}=\sigma\left(x_{i}\right)= \pm x_{i}$, and so, in $K \otimes K, m_{i}(\sigma \otimes \sigma)= \pm(\sigma \otimes \sigma) m_{i}$.

Since $K=U+\sigma U, K \otimes K$ is the vector space direct sum $U \otimes U \oplus(\sigma \otimes \sigma) U$ $\otimes U \oplus(\sigma \otimes 1) U \otimes U \oplus(1 \otimes \sigma) U \otimes U$. Since $U \otimes U=\sum \oplus m_{i} \cdot U$ as right $U$. module, $U \otimes U+(\sigma \otimes \sigma) U \otimes U=\sum \oplus m_{i} \cdot K$ is a free right $K$-module with generators $m_{i}$ : first, the $m_{i}$ are generators since $(\sigma \otimes \sigma) m_{i}= \pm m_{i}(\sigma \otimes \sigma)= \pm m_{i} \cdot \sigma$ $= \pm m_{i} D(\sigma)$, and if $\sum m_{i} \cdot k_{i}=\sum m_{i} D\left(k_{i}\right)=0$ for $k_{i} \in \bar{K}, k_{i}=a_{i}+\sigma b_{i}$ then $0=\sum m_{i} D\left(a_{i}\right) \pm(\sigma \otimes \sigma) m_{i} D\left(b_{i}\right) \in U \otimes U \oplus(\sigma \otimes \sigma)(U \otimes U)$ and so $\sum m_{i} D\left(a_{i}\right)$ $=0=\sum m_{i} D\left(b_{i}\right)$. But since the $m_{i}$ are a basis for $(U \otimes U)_{D}, D\left(a_{i}\right)=D\left(b_{i}\right)$ $=D\left(k_{i}\right)=0$. In the same way, the elements $(\sigma \otimes 1) m_{i}$ are a basis for $(\sigma \otimes 1) U$ $\otimes U \oplus(1 \otimes \sigma) U \otimes U$. Thus $(K \otimes K)_{D}$ is right $K$-free.

Thus, by [2, Chapter 10, Theorem 10.6.1] we have

TheOREM 2.1. If $A$ is any left $K^{e}$-module, so that ${ }_{E} A$ is a left $K$-module, then $\operatorname{Ext}_{K}^{n}\left(\Phi,{ }_{E} A\right)$ is isomorphic to $\operatorname{Ext}_{K}^{n}(K, A)$.

Corollary 2.1. Let $M, N$ be left $K$-modules, then $\operatorname{Ext}_{K}^{n}(M, N)$ is isomorphic to $\operatorname{Ext}_{K}^{n}\left(\Phi, \operatorname{Hom}_{\Phi}(M, N)\right)$.

$\left[\operatorname{Hom}_{\Phi}(M, N)\right.$ is a left $K$-module under $(\sigma \cdot h)(m)=\sigma h(\sigma(m))$ and $(x \cdot h)(m)$ $=x \cdot h(m)-h(x \cdot m)$ for $x \in L$.

Proof. The above definition of $H=\operatorname{Hom}_{\Phi}(M, N)$ as left $K$-module is the same as starting with $H$ as left $K^{e}$ module in the usual way: $(k \cdot h)(m)=k \cdot h(m)$, $(h \cdot k)(m)=h(k \cdot m)$ and then making it into the left $K$-module ${ }_{E} H$. Then, $\operatorname{Ext}_{K}^{n}\left(\Phi,{ }_{E} H\right)$ is isomorphic to $\operatorname{Ext}_{K^{e}}^{n}(K, H)$ and, by [2, Chapter 9, Corollary 4.4], $\operatorname{Ext}_{K^{e}}^{n}\left(K, \operatorname{Hom}_{\Phi}(M, N)\right)$ is isomorphic to $\operatorname{Ext}_{K}^{n}(M, N)$. 
Next, we note that $K$ is projective, even free, as left (or right) $U$-module, since $K=U+U \sigma=U+\sigma U$. Therefore, for any left $U$-module $A, \operatorname{Tor}_{n}^{\nabla}(K, A)$ $=0$ for $n>0$, and so, by $[2$, p. 118] we have the isomorphism

$$
\operatorname{Ext}_{K}^{n}(K \otimes U A, C) \simeq \operatorname{Ext}_{U}^{n}(A, C),
$$

for any left $K$-module $C$. In particular, let $A=\Phi$ as left $U$-module: then $K \otimes_{U} \Phi=U \otimes_{U} \Phi+\sigma U \otimes_{U} \Phi=1 \otimes_{U} \Phi+\sigma \otimes_{U} \Phi$. At this point we employ the elements $e=(1+\sigma) / 2, f=(1-\sigma) / 2$ which are orthogonal idempotents in $K$ with sum 1 ; then $K \otimes_{U} \Phi=e \otimes_{U} \Phi \oplus f \otimes_{U} \Phi$ (direct sum as left $K$-modules). $e \otimes_{U} \Phi$ is isomorphic to $\Phi$ as left $K$-module as defined before (i.e., with $\sigma$ acting as the identity) under the map $e \otimes_{U} \alpha \rightarrow \alpha$, while $\sigma\left(f \otimes_{U} \alpha\right)=-f \otimes_{U} \alpha$ (since $\sigma f=-f$ ) so that $f \otimes_{U} \Phi$, which will be denoted by $\Phi^{-}$with $f \otimes_{U} \alpha$ denoted by $\alpha$, is isomorphic to $\Phi$ as left $U$-module but $\sigma$ acts as -1 on it. Then (2.1) becomes

$$
\operatorname{Ext}_{U}^{n}(\Phi, C) \simeq \operatorname{Ext}_{K}^{n}(\Phi, C) \oplus \operatorname{Ext}_{K}^{n}\left(\Phi^{-}, C\right) \quad \text { for any left } K \text {-module } C .
$$

We now proceed to construct a specific resolution of $\Phi$ as left $K$-module. We start with the usual resolution of $\Phi$ as $U$-module.

$$
\cdots \rightarrow U \otimes \Lambda_{n} \stackrel{d_{n}}{\rightarrow} U \otimes \Lambda_{n-1} \rightarrow \cdots \rightarrow \stackrel{\epsilon}{\rightarrow} \Phi \rightarrow 0
$$

where $\Lambda$ is the exterior algebra on the vector space $L, \Lambda_{n}$ the subspace of homogeneous elements of degree $n$ of $\Lambda$, and

$$
\begin{aligned}
& d_{n}(a\left.\otimes x_{1} \wedge \cdots \wedge x_{n}\right)=\sum_{i=1}^{n}(-1)^{i+1} a x_{i} \otimes x_{1} \wedge \cdots \wedge x_{i} \wedge \cdots \wedge x_{n} \\
& \quad+\sum_{i<j}(-1)^{i+j} a \otimes\left[x_{i} x_{j}\right] \wedge x_{1} \wedge \cdots \wedge \hat{x}_{i} \wedge \cdots \wedge \hat{x}_{j} \wedge \cdots \wedge x_{n}
\end{aligned}
$$

Following the reasoning $[2$, Chapter $6, \S 4]$ leading to $(2.1)$ and $\left(2.1^{\prime}\right)$, we form the left $K$-modules $Y_{n}=K \otimes_{U}\left(U \otimes \Lambda_{n}\right) \simeq K \otimes \Lambda_{n}$ and $K \otimes_{U} \Phi \simeq \Phi \oplus \Phi^{-}$. Since $Y_{n}=K \otimes_{U} X_{n}$ and the $X_{n}$ are a $U$-projective resolution of $\Phi$ with the differentiation $d_{n}$ of (2.3), if we extend $d_{n}$ to the $Y_{n}$ by $d_{n}\left(k \otimes_{U} x_{n}\right)=k \otimes_{U}\left(d_{n} x_{n}\right)$, the $Y_{n}$ with this differentiation will be a $K$-projective resolution of $K \otimes_{U} \Phi$ (since $K$ is $U$-projective). Writing $Y_{n}$ as $K \otimes \Lambda_{n}$ we see that $d_{n}$ is given by the formula (2.3) again (but $a$, in (2.3), being now an element of $K$ ). The map $K=Y_{0} \rightarrow K \otimes_{U} \Phi$ is given by $\sigma \rightarrow \sigma \otimes_{U} 1, a \rightarrow \epsilon(a)$ for $a \in U$.

The direct sum decomposition $K \otimes_{U} \Phi=\Phi \oplus \Phi^{-}$will now be extended to a direct sum decomposition (as $K$-module) $K \otimes_{U} X_{n}=Y_{n}=Y_{n}+\oplus Y_{n}$ - such that the $Y_{n}{ }^{+}$(with differentiation induced by $d_{n}$ ) form a $K$-projective resolution of $\Phi$, and the $Y_{n^{-}}$of $\Phi^{-}$. We set

$$
\begin{aligned}
Y_{n^{+}} & =\left\{a \otimes \lambda_{n}+a \sigma \otimes \lambda_{n}^{\sigma} \mid a \in U, \lambda_{n} \in \Lambda_{n}\right\} \\
& =\left(K e \otimes \Lambda_{n}+\right) \oplus\left(K f \otimes \Lambda_{n}^{-}\right)
\end{aligned}
$$


where $\lambda \rightarrow \lambda^{\sigma}$ is the automorphism of $\Lambda$ induced by the automorphism $\sigma$ of $L$, and $\Lambda_{n}{ }^{+}=\left\{\lambda_{n} \in \Lambda_{n} \mid \lambda_{n}^{\sigma}=\lambda_{n}\right\}, \Lambda_{n}{ }^{-}=\left\{\lambda_{n} \in \Lambda_{n} \mid \lambda_{n}^{\sigma}=-\lambda_{n}\right\}$ (recall that $e=(1+\sigma) / 2, f=(1-\sigma) / 2)$. Similarly,

$$
\begin{aligned}
Y_{n^{-}} & =\left\{a \otimes \lambda_{n}-a \sigma \otimes \lambda_{n}^{*} \mid a \in U, \lambda_{n} \in \Lambda_{n}\right\} \\
& =\left(K e \otimes \Lambda_{n}-\right) \oplus\left(K f \otimes \Lambda_{n}^{+}\right) .
\end{aligned}
$$

To show that $d_{n}\left(Y_{n}{ }^{+}\right) \subset Y_{(n-1)}{ }^{+}$and $\left.d\left(Y_{n}^{-}\right) \subset Y_{(n-1)}\right)^{-}$we note that, if we write $\left(a \otimes \lambda_{n}\right) \cdot \sigma$ for $a \sigma \otimes \lambda_{n}^{\sigma}\left(a \in U, \lambda_{n} \in \Lambda_{n}\right)$ then $d_{n}$ satisfies

$$
d_{n}\left[\left(a \otimes \lambda_{n}\right) \cdot \sigma\right]=\left[d_{n}\left(a \otimes \lambda_{n}\right)\right] \cdot \sigma,
$$

therefore

$$
\begin{aligned}
d_{n}\left(a \otimes \lambda_{n}+a \sigma \otimes \lambda_{n}^{\sigma}\right) & =d_{n}\left(a \otimes \lambda_{n}+\left(a \otimes \lambda_{n}\right) \cdot \sigma\right) \\
& =d_{n}\left(a \otimes \lambda_{n}\right)+\left(d_{n}\left(a \otimes \lambda_{n}\right)\right) \cdot \sigma \in Y_{(n-1)^{+}} .
\end{aligned}
$$

The map $Y_{0}=K$ onto $K \otimes_{U} \Phi=\Phi \oplus \Phi^{-}=e \otimes_{U} \Phi \oplus f \otimes_{U} \Phi$ is given by $\sigma \rightarrow \sigma \otimes_{U} 1$, and $a \rightarrow \epsilon(a) \cdot 1$ for $a \in U$. Thus $e=(1-\sigma) / 2 \rightarrow e \otimes_{U} 1 \in \Phi$, and $f \rightarrow f \otimes_{U} 1$ in $\Phi^{-}$. This map therefore sends $Y_{0}{ }^{+}$onto $\Phi, Y_{0}-$ onto $\Phi^{-}$, and its kernel in $Y_{0}+$ is $d_{1}\left(Y_{1}^{+}\right)$and similarly for $Y_{0}^{-}$.

Finally, the $Y_{n}{ }^{+}$furnish a projective resolution of $\Phi$ as left $K$-module, and the $Y_{n}{ }^{-}$of $\Phi^{-}$.

Let now $N$ be a left $K$-module, and let

$$
C^{n}=\operatorname{Hom}_{K}\left(Y_{n}, N\right), \quad C_{+}^{n}=\operatorname{Hom}_{K}\left(Y_{n^{+}}, N\right), \quad C_{-}^{n}=\operatorname{Hom}_{K}\left(Y_{n^{-}}, N\right)
$$

so that $C^{n}=C_{+}^{n} \oplus C_{-}^{n}$. The map $d_{n}$ then induces a map $\delta^{n}: C^{n} \rightarrow C^{n+1}, C_{+}^{n} \rightarrow C_{+}^{n+1}$, $C_{-}^{n} \rightarrow C_{-}^{n+1}$. It is clear that $C^{n}$, for $n \geqq 1$, may be identified with the vector space of all multilinear alternating functions of $n$ variables in $L$ with values in $N$, while $C_{+}^{n}$ consists of those functions $f$ which satisfy the additional condition

$$
f\left(x_{1}^{\sigma}, \cdots, x_{n}^{\sigma}\right)=\sigma\left(f\left(x_{1}, \cdots, x_{n}\right)\right)
$$

and similarly the $f \in C_{-}^{n}$ satisfy $f\left(x_{1}^{\sigma}, \cdots, x_{n}^{\sigma}\right)=-\sigma\left(f\left(x_{1}, \cdots, x_{n}\right)\right)$.

We let $Z^{n}, Z_{+}^{n}, Z_{-}^{n}$ be the kernels of $\delta^{n}$ in $C^{n}, C_{+}^{n}, C_{-}^{n}$ respectively, also $B^{n}, B_{+}^{n}, B_{-}^{n}$ the images of $\delta^{n-1}$, and $H^{n}, H_{+}^{n}, H_{-}^{n}$ be the quotient spaces $Z^{n} / B^{n}$, $Z_{+}^{n} / B_{+}^{n}, Z_{-}^{n} / B_{-}^{n}$ respectively. Thus $H^{n}=H_{+}^{n} \oplus H_{-}^{n} . H_{+}^{n}$, which we shall denote also by $H_{+}^{n}(L, N)$, is thus obtained by taking those functions $f$ on $n$ variables t which are cocycles for the usual coboundary operator and satisfy in addition (2.6), modulo the coboundaries of functions satisfying (2.6) with $n-1$ for $n$ (or, equivalently, modulo those coboundaries which satisfy (2.6)).

Next we consider interpretations of the groups $H_{+}^{n}(L, N)$ for $n=0,1$ and 2.

$H_{+}^{0}=H_{+}^{0}(L, N)=\operatorname{Hom}_{K}(K e, N) / \operatorname{Hom}_{K}(\Phi, N)$ consists of the subspace of $N_{+}=e N$ of elements $n$ such that $[x, n]=0$ for all $x \in L$, i.e. the submodule of invariants of $N_{+}$. 
$H_{+}^{1}=H_{+}^{1}(L, N)$ consists of those derivations $f$ of $L$ into $N$ such that $f(\sigma(x))=\sigma(f(x))$, modulo inner derivations of the form $f(x)=[x, n]$ with $n \in N_{+}=e N$.

Another interpretation of $H_{+}^{1}$ is in terms of extensions of $K$-modules modulo inessential extensions: if $N, N^{\prime}$ are $K$-modules and $E$ an extension of $N$ by $N^{\prime}$, i.e., $0 \rightarrow N^{\prime} \rightarrow^{i} E \rightarrow^{p} N \rightarrow 0$ is exact, then the group (as defined in [2, Chapter 14]) of such extensions $E$ modulo the inessential extensions, is isomorphic to $\operatorname{Ext}_{K}^{1}\left(N, N^{\prime}\right)$, which, by Corollary 2.2, is isomorphic to $H_{+}^{1}\left(L, \operatorname{Hom}_{\Phi}\left(N, N^{\prime}\right)\right)=\operatorname{Ext}_{K}^{1}\left(\Phi, \operatorname{Hom}_{\Phi}\left(N, N^{\prime}\right)\right)$.

The group $H_{+}^{2}(L, N)$ is connected with extensions of Lie algebras with involution, i.e., extensions $0 \rightarrow N \rightarrow^{i} E \rightarrow^{p} L \rightarrow 0$ such that $N, E, L$ are all Lie algebras with involution $\sigma$ ( $N$ being an abelian ideal in $E$ ) and $i, p$ commute with $\sigma$. Such an extension $E$ yields a 2-cocycle $g \in Z_{+}^{2}(L, N)$ : choose a linear map $q$ of $L$ into $E$ such that $q \sigma=\sigma q$ and $p q(l)=l$ for $l \in L$ (a "linear inverse" to $p)$, and let $g(x, y)=[q(x), q(y)]-q([x, y])$ for $x, y \in L$. Then $g\left(x^{\sigma}, y^{\sigma}\right)$ $=\sigma g(x, y)$ and $\delta g=0$. Any other choice of a map $q$ satisfying these conditions would merely alter $g$ by the addition of an element of $B_{+}^{2}(L, N)$.

Conversely, given $g \in Z_{+}^{2}(L, N)$ one can construct an extension $E$ as the vector space direct sum $E=N \oplus L$, with multiplication

$$
\left[\left(n_{1} \oplus x\right),\left(n_{2} \oplus y\right)\right]=\left(\left[n_{1}, y\right]-\left[n_{2}, x\right]+g(x, y)\right) \oplus[x, y]
$$

and involution $\sigma(n \oplus x)=\sigma(n) \oplus \sigma(x)$. Then $E$ is a Lie algebra with involution, and the maps $i: n \rightarrow n \oplus 0, p:(n \oplus x) \rightarrow x$ of $N \rightarrow E, E \rightarrow L$, commute with $\sigma$.

One can then verify that the correspondence $g \leftrightarrow E$ gives a 1-1 correspondence between $H_{+}^{2}(L, N)$ and the equivalence classes of extensions $E$ of $L$ by $N$ (as Lie algebras with involution); the zero element of $H_{+}^{2}(L, N)$ corresponds to the class of inessential extensions $E$, i.e., those for which there is a Lie algebra homomorphism $q$ of $L$ into $N$ such that $q \sigma=\sigma q$ and $p q(l)=l$ for $l \in L$.

3. Cohomology of Lie triple systems. Let $T$ be a Lie triple system, $M$ a $T$-module. We define the $n$th cohomology group of $T$ with coefficients in $M$, $H^{n}(T, M)$ as the group $H_{+}^{n}\left(L_{u}(T), M_{s}\right)$ defined in the previous section, where $L_{u}(T)$ is the universal enveloping Lie algebra of $T$ and $M_{8}$ is the standard extension of $M$, and the involution $\sigma$ acts on $L_{u}(T)$ and on $M_{s}$ as explained in $\S 1$. Thus $H_{+}^{n}(T, M)=\operatorname{Ext}_{K}^{n}\left(\Phi, M_{s}\right)$ and is a direct summand of the ordinary $n$th cohomology group $H^{n}\left(L_{u}(T), M_{s}\right)$.

We start by examining the 0th, 1st, and 2nd cohomology groups for arbitrary $T$ and $M$, making no assumption on the dimension of $T$ and $M$ as vector spaces over the field $\Phi$ or on the characteristic of $\Phi$ (except the standing assumption that the characteristic is not two); at the end of the section we specialize to finite-dimensional semi-simple $T$ and $\Phi$ of characteristic zero, and obtain the analogues of the two Whitehead lemmas.

As shown in $\S 2, H_{+}^{0}\left(L_{u}(T), M_{s}\right)$ consists of the invariants of $\left(M_{s}\right)_{+}=e M_{s}$ $=[T, M]$ : by the definition of $M_{s}$, however, the only such invariant is zero. Thus $H^{0}(T, M)=(0)$ for all $T, M$. 
We define a derivation $D$ of $T$ into $M$ as a linear transformation satisfying

$$
D([x, y, z])=[D(x), y, z]+[x, D(y), z]+[x, y, D(z)] .
$$

$([a, b, c]$ denotes $[[a b] c]=[c[b a]]$ whenever it is permissible to use the operation $[a b]$.) Equivalently, $D$ is a derivation of $T$ into $M$ if and only if the map $h: x \rightarrow x \oplus D(x)$ of $T$ into the semi-direct sum $E=T \oplus M$ is an isomorphism into $E$.

Proposition 3.1. Every Lie triple system derivation $D$ of $T$ into $M$ can be extended uniquely to a Lie algebra derivation $D_{0}$ of $L_{u}(T)$ into $M_{*}$ such that $D_{0} \sigma=\sigma D_{0}$; conversely, every Lie algebra derivation $D_{0}$ of $L_{u}(T)$ into $M_{s}$ satisfying $D_{0} \sigma=\sigma D_{0}$ induces a Lie triple system derivation of $T$ into $M . D_{0}$ is inner if and only if $D$ has the form

$$
D(t)=\sum_{i}\left[t,\left[t_{i}, m_{i}\right]\right], \quad t_{i} \in T, m_{i} \in M .
$$

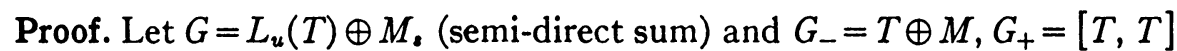
$\oplus[T, M]$ so that $G=G_{-} \oplus G_{+}$. Let $D$ be a derivation of $T$ into $M, h: t \rightarrow t \oplus D(t)$ the corresponding homomorphism of $T$ into $E=T \oplus M$. Since $L_{u}(T)$ is the universal Lie algebra of $T$ and since $h$ is a homomorphism of $T$ into the Lie algebra $G=E \oplus[T, E], h$ can be extended uniquely to a homomorphism $h_{0}$ of $L_{u}(T)$ into $G$.

Since $h(t)-t \in M$ for $t \in T$, and since $h_{0}$ is a homomorphism of Lie algebras, $h_{0}\left(\left[t_{1}, t_{2}\right]\right)-\left[t_{1}, t_{2}\right] \in[T, M]$ for $t_{i} \in T$, and so $h_{0}(l)-l \in M \oplus[T, M]=M_{\text {s }}$ for $l \in L_{u}(T)$. Thus $h_{0}(l)=l \oplus D_{0}(l) \in L_{u}(T) \oplus M_{s}$ and $D_{0}$ is a derivation of $L_{u}(T)$ into $M_{8}$ which agrees with $D$ on $T$. Since $D_{0}(T) \subseteq M, D_{0}([T, T]) \subseteq[T, M]$, and $\sigma D_{0}=D_{0} \sigma . D_{0}$ is uniquely determined by $D$ since $T$ generates $L_{u}(T)$. Conversely, it is clear that any $D_{0}$ satisfying $D_{0} \sigma=\sigma D_{0}$ maps $T$ into $M$ and so induces a Lie triple system derivation of $T$ into $M$.

Finally, let $D_{0}$ be inner: $D_{0}(l)=[l, n]$ where $n=n_{-} \oplus n_{+} \in M \oplus[T, M]$. Taking $l=t \in T$ and using the fact that $D_{0} \sigma=\sigma D_{0}$, we find that $\left[t, n_{-}\right]=0$ and $D_{0}(t)=\left[t, n_{+}\right]$(so that $D_{0}(l)=\left[l, n_{+}\right]$for all $l \in L_{u}(T)$ also). But $n_{+}$, being in $[T, M]$, has the form $\sum_{i}\left[t_{i}, m_{i}\right]$, and so $D(t)=D_{0}(t)$ satisfies (3.2). The converse is clear. Thus the proof is complete.

A Lie triple system derivation is called inner if it satisfies (3.2). Thus Proposition 3.1, together with the considerations of $\$ 2$, yields

THEOREM 3.1. The group $H^{1}(T, M)$ is isomorphic to the group of derivations of $T$ into $M$ modulo the subgroup of inner derivations.

Next we consider algebra extensions and the second cohomology group.

Let $E, T$ be Lie triple systems, $p$ a homomorphism of $E$ onto $T$ with kernel $M$ : thus $0 \rightarrow M \rightarrow^{i} E \rightarrow^{p} T \rightarrow 0$ is exact, $i$ denoting the injection. We shall always assume that, if $x, y, z \in E,[x, y, z]=0$ if any two of $x, y, z$ are in $M$ : then $M$ is a $T$-module in the natural way. All such extensions $E$ of $T$ by $M$ 
( $M$ being a $T$-module) may be cons ${ }^{+-}$, cted by means of factor sets $f(x, y, z)$, which are alternating tri-linear maps of $T \times T \times T$ into $M$ satisfying certain i.lentities: as vector space $E=M \oplus T$, and the multiplication in $E$ is given by

$$
\left[\left(0 \oplus t_{1}\right),\left(0 \oplus t_{2}\right),\left(0 \oplus t_{3}\right)\right]=f\left(t_{1}, t_{2}, t_{3}\right) \oplus\left[t_{1}, t_{2}, t_{3}\right]
$$

together with the $T$-module structure of $M$. Thus $f$ has to satisfy certain identities obtained from the multilin ar identities (1.1)-(1.3); further, each of these identities consists of sums of monomials which are of first degree in $f$, so that the factor sets form a vector space over the base field. We shall show that the group (actually vector space) of factor sets modulo the subgroup (subspace) of trivial factor sets is isonorphic to the second cohomology group $H^{2}(T, M)$ : the trivial factor sets are defined to be those associated with inessential extensions $E$, i.e., those extensions for which there exists a Lie triple system homomorphism $q$ of $T$ into $E$ such that $p q(t)=t$ for $t \in T$; equivalently, a factor set $f$ is trivial if there is a linear map $h$ of $T$ into $M$ such that

$$
f\left(t_{1}, t_{2}, t_{3}\right)=h\left(\left[t_{1}, t_{2}, t_{3}\right]\right)-\left[i\left(t_{1}\right), t_{2}, t_{3}\right]+\left[h\left(t_{2}\right), t_{1}, t_{8}\right]-\left[t_{1}, t_{2}, h\left(t_{8}\right)\right]
$$
(see [9]).

THEOREM 3.2. The group $H^{2}(T, M)$ is isomorphic to the group of factor sets of $T$ into $M$ modulo the subgroup of trivial factor sets.

Proof. We shall associate extensions of Lie triple systems to extensions of Lie algebras with involution, and then use the correspondence between the latter and elements of $H_{+}^{2}\left(L_{u}(T), M_{\varepsilon}\right)=H^{2}(T, M)$ discussed in $\S 2$. The main step is:

Lemma. Let $0 \rightarrow M \rightarrow^{i} E \rightarrow^{p} T \rightarrow 0$ be an extension of $T$ by $M$. Then there exists an extension of Lie algebras with involution $0 \rightarrow M_{s} \rightarrow i_{0} G \rightarrow p_{0} L_{u}(T) \rightarrow 0$ of $L_{u}(T)$ by $M_{s}$ such that the following diagram is commutative ( $j$ denoting injections):

$$
\begin{aligned}
& 0 \rightarrow M \stackrel{i}{\rightarrow} E \stackrel{p}{\rightarrow} T \rightarrow 0 \\
& \downarrow j \quad \downarrow j \quad \downarrow j \\
& 0 \rightarrow M_{\imath} \rightarrow G \rightarrow L_{u}(T) \rightarrow 0 .
\end{aligned}
$$

Proof. The homomorphism $p$ of $E$ onto $T$ induces a homomorphism $p^{\prime}$ of $L_{u}(E)$ onto $L_{u}(T)$ which commutes with $\sigma$. We show first that the kernel of $p^{\prime}$ is the ideal $I=M+[E, M]$ in $L_{u}(E)$ generated by $M$. We will then define $G$ as the quotient of $L_{u}(T)$ by another ideal $J \subset I$ such that $I / J$ is isomorphic to $M$.

It is clear that $I=M+[E, M] \subset$ kernel of $p^{\prime}$ since $M C$ kernel of $p^{\prime}$. On the other hand, the natural homomorphism $r$ of $L_{u}(E)$ onto $L_{u}(E) / I$ induces a homomorphism of $E$ into $L_{u}(E) / I$ and maps $M$ into zero, thus $r$ can be factored through $L_{u}(T)$, 


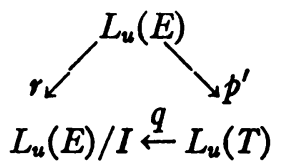

$r=q p^{\prime}$. Thus kernel of $p^{\prime} \subset$ kernel of $r=I$, and so kernel of $p^{\prime}=I$. (Since $\sigma(I)=I, \sigma$ also acts on $L_{u}(E) / I$ so that $r \sigma=\sigma r$. Since $p^{\prime} \sigma=\sigma p^{\prime}$ and $p^{\prime}$ is onto, $q \sigma=\sigma q$ also.)

We now define the ideal $J$ in $L_{u}(E)$ as $J=\{x \in[E, M] \mid[x, a]=0$ for all $a \in E\}$. Since $[J, E]=0$ and $E$ generates $L_{u}(E),\left[J, L_{u}(E)\right]=0$. Also, $\sigma(x)=x$ for all $x \in J$, and so $J \cap E=(0)$.

Let $G=L_{u}(E) / J$, and $j$ the natural homomorphism of $L_{u}(E)$ onto $G . j$ is 1-1 on $E$, and so may be regarded as an inclusion map on $E$. $G$ is also a Lie algebra with involution $\sigma$ and $\sigma j=j \sigma$, since $\sigma(J)=J$ in $L_{u}(E)$. Since $J C I$, the projection $p^{\prime}$ of $L_{u}(E)$ onto $L_{u}(T)$ induces a homomorphism $p_{0}$ (also commuting with $\sigma)$ of $G$ onto $L_{u}(T): p_{0} j=p$ on $L_{u}(E) . J \supset[M, M]$ since $[x, y, z]=0$ in $E$ whenever two of $x, y, z$ are in $M$, so $[j(M), j(M)]=0$ in $G$. The kernel $N$ of $p_{0}$ is $j(I)=j(M \oplus[E, M])=j(M) \oplus[j(E), j(M)]$.

Let $q$ be a 1-1 linear map of $T \rightarrow E$ such that $p q(t)=t$ for $t \in T$, then $E=M \oplus q(T)$ as vector space, so $j(E)=j(M) \oplus j q(T)$. The kernel of $p_{0}$ may then be written as $N=j(M) \oplus[j(E), j(M)]=j(M) \oplus[j q(T), j(M)]$, since $[j(M), j(M)]=0$.

$N$ may be considered as $L_{u}(T)$ module by $[t, n]=[j q(t), n]$ (this is independent of the choice of $q$ since $[j(M), j(M)]=0$ and [ $\left.[j q(t), j(m)], j\left(m^{\prime}\right)\right]$ $\left.=j\left(\left[q(t), m, m^{\prime}\right]\right)=0\right)$. The induced structure on $j(M)$ as Lie triple system module for $T$ is then isomorphic to $M$ as $T$-module, under the map $m \rightarrow j(m):\left[j(m), j q(t), j q\left(t^{\prime}\right)\right]=j\left(\left[m, t, t^{\prime}\right]\right)$. We have to show $N$ is isomorphic to $M_{s}$ as $L_{u}(T)$ module.

Since $N=j(M) \oplus[j q(T), j(M)]=j(M) \oplus[T, j(M)]$, there is an $L_{u}(T)$ homomorphism of $N$ onto $M$, taking $j(m) \rightarrow m,[j(m), t] \rightarrow[m, t]$. To show this map is $1-1$, we suppose that $\sum\left[j\left(m_{i}\right), t_{i}\right] \rightarrow \sum\left[m_{i}, t_{i}\right]=0$ (the map being known to be $1-1$ on $j(M))$. Then $\sum\left[m_{i}, t_{i}, t\right]=0$ in $M$ for all $t \in T$, and, in $E, \sum\left[m_{i}, q\left(t_{i}\right), q(t)\right]=0$. Thus $\sum\left[m_{i}, q\left(t_{i}\right)\right] \in J$ (since $\sum\left[\left[m_{i}, q\left(t_{i}\right)\right], m\right]=0$ automatically for $m \in M)$, and, applying $j, \sum\left[j\left(m_{i}\right), j q\left(t_{i}\right)\right]=\sum\left[j\left(m_{i}\right), t_{i}\right]=0$ in $G$.

Finally, it is clear that the given diagram is commutative if $j$ also denotes the inclusion of $T$ into $L_{u}(T)$. This concludes the proof of the lemma.

To each factor set $g$ of $L_{u}(T)$ into $M$, satisfying $g(\sigma(x), \sigma(y))=\sigma g(x, y)$ (i.e., element of $Z_{+}^{2}\left(L_{u}(T), M_{s}\right)$ ) we shall assign a factor set $f=\alpha(g)$ as follows: from $g$ we construct an extension $G$ of $L_{u}(T)$ by $M_{\text {, as }}$ in the last section: $G=M_{\bullet} \oplus L_{u}(T)$ as vector space, the involution is $\sigma(n \oplus x)=\sigma(n) \oplus \sigma(x)$, and the multiplication is determined by: $[0 \oplus x, 0 \oplus y]=g(x, y) \oplus[x, y]$. Thus $E=M \oplus T \subset G$. We then have the exact sequence $0 \rightarrow M_{0} \rightarrow^{i} G \rightarrow{ }^{p} L_{u}(T) \rightarrow 0$, and (by restricting the map $i$ to $M, p$ to $E=T \oplus M$ ) the exact sequence $0 \rightarrow M \rightarrow^{i} E$ 
$\rightarrow^{p} T \rightarrow 0$, i.e., we also have an extension $E$ of $T$ by $M$. The multiplication in $E=M \oplus T$ is determined by

$$
\left[\left[0 \oplus t_{1}, 0 \oplus t_{2}\right], 0 \oplus t_{3}\right]:=f\left(t_{1}, t_{2}, t_{3}\right) \oplus\left[\left[t_{1}, t_{2}\right], t_{3}\right]
$$

where

$$
f\left(t_{1}, t_{2}, t_{3}\right)=\left[g\left(t_{1}, t_{2}\right), t_{3}\right]+g\left(\left[t_{1}, t_{2}\right], t_{3}\right) .
$$

In short, $f=\alpha(g)$ is determined by (3.6). It is then clear that $\alpha$ is a linear map of $Z_{+}^{2}\left(L_{u}(T), M_{s}\right)$ into the space $F$ of factor sets $f$ of $T$ into $M$.

If the factor set $g$ is trivial, i.e., $\in B_{+}^{2}\left(L_{u}(T), M_{s}\right)$ then, in terms of the extension $G \rightarrow{ }^{p} L_{u}(T)$, there is a Lie algebra homomorphism $q$ of $L_{u}(T)$ into $G$ such that $p q(l)=l$ for $l \in L_{u}(T)$, and $q \sigma=\sigma q$. Thus $q$ maps $T$ into $E$ and so the factor set $f$ is also trivial. Explicitly, if $q(l)=h(l) \oplus l$ where $h \in C_{+}^{1}\left(L_{u}(T), M_{\text {s }}\right)$ then $f$ satisfies (3.4) with this function $h$.

Conversely, suppose $f=\alpha(g)$ and $f$ is trivial. Using the extensions $G$ of $L_{u}(T)$ by $M_{s}$ and $E$ of $T$ by $M$ just described, we have a homomorphism of Lie triple systems $q$ of $T$ into $E$ such that $p q(t)=t$ for $t \in T$. Since $L_{u}(T)$ is the universal Lie algebra of $T$, and $q$ is a homomorphism of $T$ into the Lie algebra $G, q$ can be extended to a homomorphism $q_{0}$ of $L_{u}(T)$ into $G$. Since $q_{0}(T) \subset E, q_{0}([T, T]) \subset[E, E]$ and so $q_{0} \sigma=\sigma q_{0}$. Finally, $p q_{0}(t)=t$ for $t \in T$, and so $p q_{0}$ is the identity on $L_{u}(T)$. Thus $g$ is also a trivial factor set.

Finally, the map $\alpha$ is onto $F$ : given $f \in F$, we construct an extension $E$ of $T$ by $M$ with this factor set, i.e., we find a linear inverse $q$ of $p$ such that $f\left(t_{1}, t_{2}, t_{3}\right)$ $\left.=\left[\left[q\left(t_{1}\right), q\left(t_{2}\right)\right], q\left(t_{3}\right)\right]-q\left(\left[t_{1}, t_{2}\right], t_{3}\right]\right)$. By the lemma, we can construct a corresponding extension $G$ of $L_{u}(T)$ by $M_{s}$ such that the projection $p_{0}$ of $G$ onto $L_{u}(T)$ agrees with $p$ on $E$. Then $q$ can be extended to a linear inverse $q_{0}$ of $p_{0}$, mapping $T$ into $E,[T, T]$ into $[E, E]$. If $g(x, y)=\left[q_{0}(x), q_{0}(y)\right]-q_{0}([x, y])$ then $f\left(t_{1}, t_{2}, t_{3}\right)=\left[g\left(t_{1}, t_{2}\right), t_{3}\right]+g\left(\left[t_{1}, t_{2}\right], t_{3}\right)$ so $f=\alpha(g)$. Thus $\alpha$ is an isomorphism of $H_{+}^{2}\left(L_{u}(T), M_{s}\right)$ onto the group of factor sets of $T$ into $M$ modulo trivial factor sets.

For the rest of this section we shall specialize to $\Phi$ of characteristic zero and finite-dimensional $T$ and $M$. The radical of $T$ can then be defined (see [9]) as the maximal solvable ideal (an ideal $I$ being a subspace such that $[I, T, T] \subset I$, and solvable meaning that the series $I^{(0)}=I, I^{(n+1)}=\left[T, I^{(n)}, I^{(n)}\right]$ terminates with zero). If the radical is zero, $T$ is called semi-simple, and this condition is exactly equivalent to $L_{u}(T)$ being semi-simple. If $T$ is semisimple, it is a direct sum of simple ideals $T_{i}$ and $L_{u}(T)$ is the direct sum of the $L_{u}\left(T_{i}\right)$. If $T$ is simple, then either $L_{u}(T)$ is a simple Lie algebra and $T$ is not isomorphic to a Lie algebra considered as Lie triple system, or else $T$ is isomorphic to a simple Lie algebra $L$ considered as Lie triple system and $L_{u}(T)=L \oplus L$ with involution $\sigma(a \oplus b)=b \oplus a$ : in other words, $L_{u}(T)$ is simple when considered as Lie algebra with involution, i.e., it has no proper selfadjoint ideals. 
THEOREM 3.3. Let $T$ be a finite-dimensional semi-simple Lie triple system over a field of characteristic zero, $M$ a finite-dimensional module. Then $H^{1}(T, M)$ $=0=H^{2}(T, M)$.

Proof. $L_{u}(T)$ is finite-dimensional and semi-simple and $M_{8}$ is finitedimensional, thus $H^{1}\left(L_{u}(T), M_{s}\right)=0=H^{2}\left(L_{u}(T), M_{s}\right)$. But $H_{+}^{n}\left(L_{u}(T), M_{s}\right)$ $=H^{n}(T, M)$ is a direct summand of $H^{n}\left(L_{u}(T), M_{s}\right)$, and so is also zero for $n=1,2$.

COROLlaRY. Under the same hypotheses as in Theorem 3.3, every derivation of $T$ into $M$ is inner, and every factor set of $T$ into $M$ is trivial.

As shown in [9], the statement on factor sets for semi-simple $T$ can be used to prove the Levi decomposition for any finite dimensional Lie triple system $E: E=T+R, T$ semi-simple, $R$ the radical. The Malcev-HarishChandra uniqueness theorem for the Levi decomposition is easy to prove: it states that, if $E=T_{1}+R=T_{2}+R, T_{i}$ semi-simple, then $T_{1}$ is carried onto $T_{2}$ by an automorphism of $E$ of the form $\exp (\operatorname{Ad} z), z \in[E, R] \subset$ radical of $L_{u}(E)$, i.e., the automorphism is

$$
x \rightarrow x+\sum_{i}\left[u_{i}, v_{i}, x\right]+\frac{1}{2} \sum_{i, j}\left[u_{i}, v_{i},\left(\left[u_{j}, v_{j}, x\right]\right)+\cdots \text { for } u_{i} \in E, v_{i} \in R .\right.
$$

Such automorphisms form a group since $[E, R]$ is a subalgebra of $L_{u}(E)$.

Proposition 3.2. If $T$ is finite-dimensional semi-simple over a field of characteristic zero, then every finite-dimensional module $M$ is completely reducible.

Proof. Let $M^{\prime}$ be a submodule of $M$; then $M_{s}^{\prime}$ can be considered as a submodule of $M_{s}$ and is mapped into itself by $\sigma$, so that the factor module $P=M_{s} / M_{s}^{\prime}$ can be also considered as module with involution for $L_{u}(T)$. The sequence of $K$-modules $0 \rightarrow M_{s}^{\prime} \rightarrow{ }^{i 0} M_{s} \rightarrow P \rightarrow 0$ splits, since

$$
\operatorname{Ext}_{K}^{1}\left(P, M_{s}\right) \simeq H_{+}^{1}\left(L_{u}(T), \operatorname{Hom}_{\Phi}\left(P, M_{s}\right)\right)=0,
$$

and so there is a homomorphism of $M_{8}$ onto $M_{s}^{\prime}$ inverse to $i_{0}$ and commuting with $\sigma$. This homomorphism maps $M$ onto $M^{\prime}$, so $M^{\prime}$ has a complement in $M$.

We can say something about the third cohomology group in case the module $M=\Phi$ (with the elements of $T$ being zero-operators) so that $M_{s}=\Phi$ also:

THEOREM 3.4. Let $T$ be simple and finite-dimensional over an algebraically closed field $\Phi$ of characteristic zero. Then $H^{3}(T, \Phi)$ is either zero or one-dimensional, according as $L_{u}(T)$ is simple or not.

Proof. If $L_{u}(T)$ is simple, then $H^{3}\left(L_{u}(T), \Phi\right)$ is one-dimensional and spanned by the class of the cocycle $f(x, y, z)=B([x, y], z)$ where $B(x, y)$ is the invariant symmetric bilinear form $\operatorname{tr}(\operatorname{Ad} x \operatorname{Ad} y)$ on $L_{u}(T)$ (see [8]). Since $\sigma$ is an automorphism, $\operatorname{Ad}(\sigma x)=\sigma \cdot \operatorname{Ad} x \cdot \sigma^{-1}$ so $B(\sigma x, \sigma y)=B(x, y)$ and 
$f(\sigma x, \sigma y, \sigma z)=f(x, y, z)=-\sigma f(x, y, z)$, so that $f \in Z_{-}^{3}\left(L_{u}(T), \Phi\right)$ and $H_{+}^{3}\left(L_{u}(T), \Phi\right)$ $=0$.

Now suppose $L_{u}(T)$ is not simple, and so is the direct sum $L_{1} \oplus L_{2}$ of simple ideals mapped onto one another by $\sigma$. Denote $L_{u}(T)$ by $L$. Then the exterior algebra $\Lambda(L) \simeq \Lambda\left(L_{1}\right) \otimes \Lambda\left(L_{2}\right)$ (tensor product of anti-commutative graded algebras), and so the dual space $\Lambda^{*}(L) \simeq \Lambda^{*}\left(L_{1}\right) \otimes \Lambda^{*}\left(L_{2}\right)$. The cohomology algebra $H(L) \simeq H\left(L_{1}\right) \otimes H\left(L_{2}\right)$. Since the $L_{i}$ are simple, $H^{n}\left(L_{i}\right)=H^{n}\left(L_{i}, \Phi\right)$ is zero-dimensional for $n=1,2$, and one-dimensional for $n=0,3$. Thus $H^{3}(L)$ $=H^{3}(L, \Phi)$ is two-dimensional, spanned by the cohomology classes of $f_{1} \otimes 1^{*}$ $+1^{*} \otimes f_{2}$ and $f_{1} \otimes 1^{*}-1^{*} \otimes f_{2}$ where the $f_{i}$ are the 3-cocycles of $L_{i}$ described above and chosen so that $f_{1}(\lambda)=f_{2}(\sigma \lambda)$ for $\lambda \in \Lambda^{3}\left(L_{1}\right)$. Thus for $\lambda \in \Lambda^{3}\left(L_{1}\right)$, $\sigma \lambda \in \Lambda^{3}\left(L_{2}\right)$, and $\left(f_{1} \otimes 1^{*}+1^{*} \otimes f_{2}\right)(\sigma \lambda)=f_{2}(\sigma \lambda)=f_{1}(\lambda)=\left(f_{1} \otimes 1^{*}+1^{*} \otimes f_{1}\right)(\lambda)$ so $f_{1} \otimes 1^{*}+1^{*} \otimes f_{2} \in Z_{-}^{3}$, and similarly $f_{1} \otimes 1^{*}-1^{*} \otimes f_{2} \in Z_{+}^{3}$. Thus $H^{3}(T, \Phi)$ $=H_{+}^{3}\left(L_{u}(T), \Phi\right)$ is one-dimensional.

\section{BiBLIOGRAPHY}

1. E. Cartan, Oeuvres complètes, Part 1, vol. 2, nos. 101, 138, Paris, Gauthier-Villars, 1952.

2. H. Cartan and S. Eilenberg, Homological algebra, Princeton, N. J., Princeton University Press, 1956.

3. C. Chevalley and S. Eilenberg, Cohomology theory of Lie groups and Lie algebras, Trans. Amer. Math. Soc. vol. 63 (1948) pp. 85-124.

4. S. Eilenberg, Extensions of general algebras, Annales de la Société Polonaise de Mathematique vol. 21 (1948) pp. 125-134.

5. N. Jacobson, Structure of alternative and Jordan bimodules, Osaka Math. J. vol. 6 (1954) pp. $1-71$.

6. - Lie and Jordan triple systems, Amer. J. Math. vol. 71 (1949) pp. 149-170.

7. - General representation theory of Jordan algebras, Trans. Amer. Math. Soc. vol. 70 (1951) pp. 509-548.

8. J. L. Koszul, Homologie et cohomologie des algèbres de Lie, Bull. Soc. Math. France vol. 78 (1950) pp. 65-127.

9. W. G. Lister, A structure theory for Lie triple systems, Trans. Amer. Math. Soc. vol. 72 (1952) pp. 217-242.

10. G. D. Mostow, Decomposition theorems for semi-simple Lie groups, Memoirs Amer. Math. Soc. no. 14, 1955.

11. Séminaire Sophus Lie, Ecole Normale Supérieure, 1954-1955.

12. B. Harris, Derivations of Jordan algebras, Pacific J. Math. vol. 9 (1959) pp. 495-512.

13. E. J. Taft, Invariant Wedderburn factors, Illinois J. Math. vol. 1 (1957) pp. 565-573.

14. K. Yamaguti, On algebras of totally geodesic spaces (Lie triple systems), J. Sci. Hiroshima Univ. Ser. A vol. 21 (1957-1958) pp. 107-113.

15. - On the Lie triple system and its generalization, J. Sci. Hiroshima Univ. Ser. A vol. 21 (1957-1958) pp. 155-159.

16. - A note on a theorem of N. Jacobson, J. Sci. Hiroshima Univ. Ser. A vol. 22 (1958) pp. 187-190.

NORTHWESTERN UNIVERSITY, Evanston, IlLinoIS 